\title{
Primary squamous cell carcinoma of the liver: A case report and review of the literature
}

\author{
RONGRONG ZHAO $^{1 *}$, KUNLI ZHU $^{1 *}$, RENBEN WANG $^{1}$, JIE GAO $^{1}$, \\ KAI CUI ${ }^{2}$, FACHANG YU ${ }^{3}$, BO ZHANG ${ }^{1}$ and SHENG LI ${ }^{1}$ \\ ${ }^{1}$ Department of Hepatobiliary Surgery, Shandong Cancer Hospital, Jinan 276000; \\ ${ }^{2}$ Key Laboratory for Rare and Uncommon Diseases of Shandong Province, Jinan 250062; \\ ${ }^{3}$ Shandong Academy of Medical Sciences, Jinan 250014, P.R. China
}

Received April 4, 2012; Accepted July 19, 2012

DOI: $10.3892 / 01.2012 .894$

\begin{abstract}
Primary squamous cell carcinoma of the liver is rare. It has a high malignancy rate and poor prognosis, and survival is typically no longer than one year. The pathogenesis of the disease is unclear, but is generally considered to be correlated with the long-term inflammation or metaplasia of biliary epithelial cells and congenital cyst of the liver. The current viewpoint is that surgical resection of the tumor, preoperative or postoperative subsidiary chemoembolization, radiotherapy and generalized chemotherapy may extend a patient's survival. In the present case study, a patient suffered from biliary calculosis for over 20 years. We theorized that the mechanism of carcinomatous change was correlated with the persistent stimulation of chronic biliary infection induced by calculus of the intrahepatic duct. However, the transformation mechanism from cholangiocellular carcinoma to squamous carcinoma is undetermined and further studies are needed.
\end{abstract}

\section{Introduction}

Primary squamous cell carcinoma of the liver is rare. More than 20 cases have been reported since the first case was described in 1934 by Imai (1). The pathogenesis of the disease is undetermined. Previous studies have reported that potentially related diseases, including congenital cyst of the liver (2-8), calculus of the intrahepatic duct (9), hepatic cirrhosis $(10,11)$, Caroli's disease (12) and hepatic teratoma (1), are rare. However, hepatic primary squamous carcinoma without latent hepatic injury has also been reported (13). This disease has a high malignancy rate and poor prognosis, and survival is typi-

Correspondence to: Dr Sheng Li, Department of Hepatobiliary Surgery, Shandong Cancer Hospital, Jiyan Road 440, Jinan 276000, P.R. China

E-mail: drlisheng@sohu.com

${ }^{*}$ Contributed equally

Key words: liver primary squamous cell carcinoma cally no longer than one year (12). In the present case study, a patient suffered from biliary calculosis for over 20 years. The patient underwent chest, abdominal and pelvic CT scans and gynecological examination to preclude other primary foci. The study was approved by the Ethics Committee of Shandong Cancer Hospital, Jinan, China. Written informed consent was obtained from the patient. The postoperative pathology and immunohistochemistry confirmed the disease as liver primary squamous cell carcinoma. Combined with the patient's history of calculosis of the biliary tree for 20 years, we determined that the mechanism of carcinogenesis was correlated with persistent stimulation induced by chronic biliary infection caused by calculus of the intrahepatic duct. However, the transformation mechanism from cholangiocellular carcinoma to squamous carcinoma is undetermined and requires further study. In the present study, the patient underwent liver tumor radiofrequency ablation, but the abdominal pain remained evident following the surgery. As the patient's physical condition was too weak to tolerate generalized chemotherapy, local radiotherapy and supportive therapy is being conducted, and the patient's pain has already been relieved.

\section{Case report}

A 60-year-old female patient underwent cholecystectomy due to cholecystolithiasis with chronic cholecystitis in 1988. In May 2010, the patient experienced repeated pain without obvious cause in the right upper quadrant, accompanied by fever, nausea without vomiting, hypodynamia, anorexia, abdominal distension, diarrhea, shivers, jaundice and lumbar-dorsal radiating pain. A CT examination revealed a calculus of the intrahepatic duct and chronic cholangitis. A partial resection of the hepatic left lobe was carried out on July 29th, 2010. The postoperative pathology indicated left hepatic cholangeitis and calculus of the bile duct. A magnetic resonance imaging (MRI) examination performed in December 2010 indicated that the primary focus was in the left liver at a size of $4.0 \times 3.0 \times 3.0 \mathrm{~cm}$, with reinforcement in the arterial phase. The examination of tumor biomarkers revealed $7.6 \mu \mathrm{g} / \mathrm{l}$ AFP, $1.1 \mu \mathrm{g} / \mathrm{l}$ CEA and $275.9 \mathrm{U} / \mathrm{ml}$ CA19-9. The tumor resection of the left hepatic external lobe, exploration of the biliary tract and T-tube drainage 

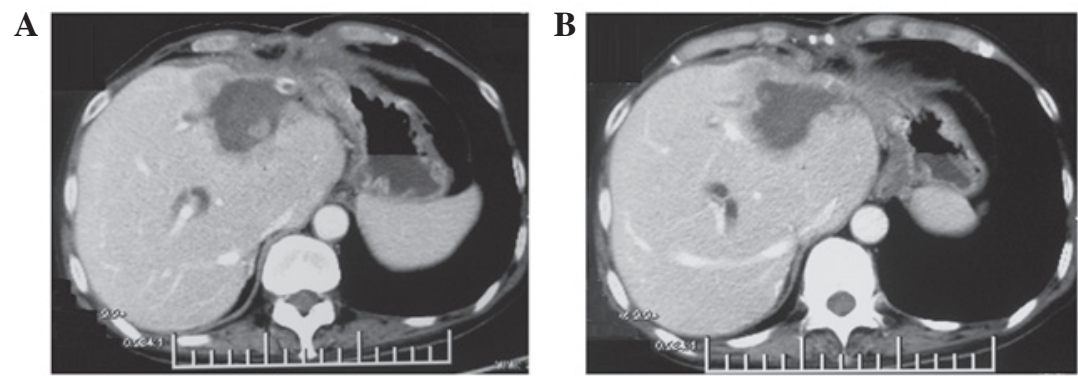

Figure 1. Abdominal CT examination. An abnormal density shadow in the medial segment of the left lobe of the liver following the tumor resection of the hepatic left lobe, which was considered to be the postoperative recurrence of cholangiocellular carcinoma of the hepatic left lobe.

were performed on December 31st, 2010. A calculus with a diameter of $0.2 \mathrm{~cm}$ and a grey tumor $2.0 \times 1.5 \mathrm{~cm}$ in the cross section without peplos in the surroundings of the tumor were observed and the texture was hard. Microscopically (Fig. 2), the tumor tissue was accumulated in the glandular tube, the lumens contained mucilage, the cancer cells were cubic or columnar, the nuclei were round or orbicular-ovate, there was karyomegaly and anachromasis, evident atypia and the surroundings indicated infiltrative growth affecting the nerves. Tumor growth was not observed in cancerous tissue, and there was no pseudo-lobule structure in the remaining liver. Masson (+), AB (+) and reticulum staining (-) were performed. The immunohistochemistry results were as follows (Fig. 3): Hep-1(+++), HBsAg(-), CK18(+), CK19(++), CD34(-), HbeAg(-), pCEA(+++), $\beta$-catenin(-), MUC-1(-), myoglobin(-), Gly-3(-) and MAT1(-). The pathological diagnosis was intrahepatic cholangiocarcinoma of the hepatic left lobe with mild differentiation and calculus of the bile duct. The patient's symptoms were relieved following the surgery. In June 2011, the patient experienced pain in the right upper quadrant without any evident cause, and the pain started to radiate to the back, accompanied by nausea, vomiting, hypodynamia and anorexia. An abdominal CT examination (Fig. 1) was performed and revealed an abnormal density shadow in the medial segment of the left lobe of the liver following the tumor resection of the hepatic left lobe, which was considered to be the postoperative recurrence of cholangiocellular carcinoma of the hepatic left lobe. Examination of the tumor markers indicated $4.63 \mu \mathrm{g} / 1 \mathrm{AFP}, 191.5 \mathrm{U} / \mathrm{ml} \mathrm{CA} 125$ and 1557.8 U/ml CA19-9. The patient underwent hepatic tumor biopsy and hepatic tumor radiofrequency ablation on July 4th, 2011. The tumor was located in the junctional zone of the hepatic left internal lobe and right anterior lobe with a hard texture at a size of $1.5 \times 1.2 \times 0.5 \mathrm{~cm}$, which was closely adhered to partial gastric serous tunic and peripheral abdominal wall. Considering the extra hepatic encroachment of the tumor, the location and surroundings of the tumor made surgical separation and radical resection difficult; therefore, radiofrequency ablation of the left hepatic tumor was carried out. Postoperative pathology revealed a mass of grayish-white and grayish-yellow tissue with a hard texture and a size of $1.2 \times 1.2 \times 0.4 \mathrm{~cm}$. Microscopically, infiltration of poorly differentiated carcinoma tissue in the proliferative fibrous tissue and interaction between foam cells and multinucleated giant cells in the mesenchyme were observed (Fig. 4). The immunohistochemical staining of the tumor cells revealed CD68(-),
Ki-67(+, <25\%), CK(+), CK590(+) (Fig. 5), which is consistent with liver squamous cell carcinoma.

Currently, the patient continues to feel pain in the right upper quadrant of the abdomen, accompanied by fever, nausea, vomiting, hypodynamia, anorexia without jaundice, abdominal distension and diarrhea, with poor diet and sleeping. The patient's weight has reduced by $\sim 15 \mathrm{~kg}$ in 1 year. Nutritional therapy was administered, and radiotherapy of the hepatic tumor focus and the periphery of the invaded structure were performed at a total dose of $36 \mathrm{~Gy} 20$ times. The patient's abdominal pain was markedly relieved and the abdominal mass was reduced to a size of $4.0 \times 5.0 \mathrm{~cm}$, and we are currently following up the patient.

\section{Discussion}

Incidence of primary squamous cell carcinoma of the liver is rare and the pathogenesis of the disease is undetermined. Previous studies have reported that potentially related diseases, including congenital cyst of the liver $(7,8)$, calculus of the intrahepatic duct (9), hepatic cirrhosis (11), Caroli's disease (12) and hepatic teratoma (1), are also rare.

In the present study, the patient underwent chest, abdominal and pelvic CT scans and gynecological examination to preclude other primary foci. The postoperative pathology and immunohistochemistry confirmed the disease as liver primary squamous cell carcinoma. The patient's primary operative pathology revealed hepatic cholangiocellular carcinoma. The tumor recurrence occurred in half a year, and tumor pathological biopsy and immunohistochemistry revealed the tumor to be hepatic primary squamous carcinoma. The transformation from hepatic cholangiocellular carcinoma to liver squamous carcinoma is extremely rare, and there are no similar studies. Combined with the patient's history of calculosis of the biliary tree for 20 years, we determined that the mechanism of carcinogenesis was correlated with persistent stimulation induced by chronic biliary infection caused by calculus of the intrahepatic duct. However, the transformation mechanism from cholangiocellular carcinoma to squamous carcinoma is undetermined and requires further study.

The morbidity of hepatic primary squamous has no evident gender differentiation and mainly occurs in 50-70-year-old patients. The clinical symptoms are commonly reported as stomach ache or jaundice, accompanied by anorexia, hypodynamia, nausea, vomiting and physical athrepsia. Swelling of the liver may be palpable in the physical examination. 

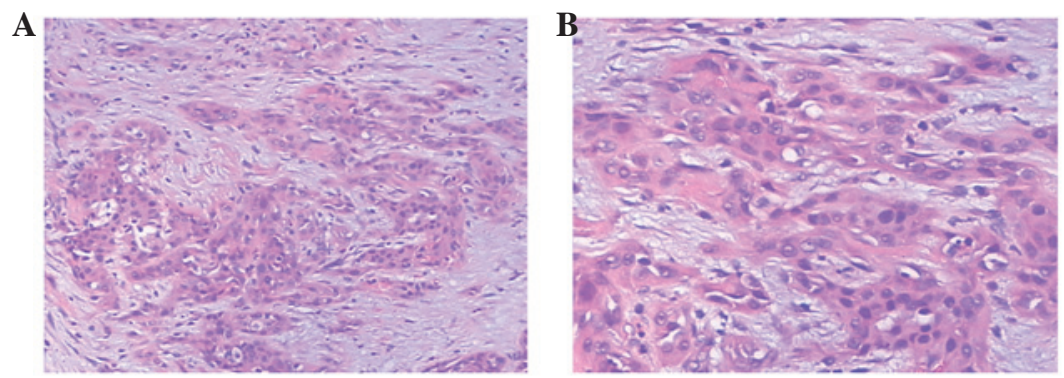

Figure 2. Microscopically, the tumor tissue accumulated in the glandular tube. The cancer cells were cubic or columnar, the nuclei were round or orbicularovate, and there was karyomegaly, anachromasis and evident atypia. (A) H\&E staining; magnification, x100. (B) H\&E staining; magnification, x200. H\&E hematoxylin and eosin.
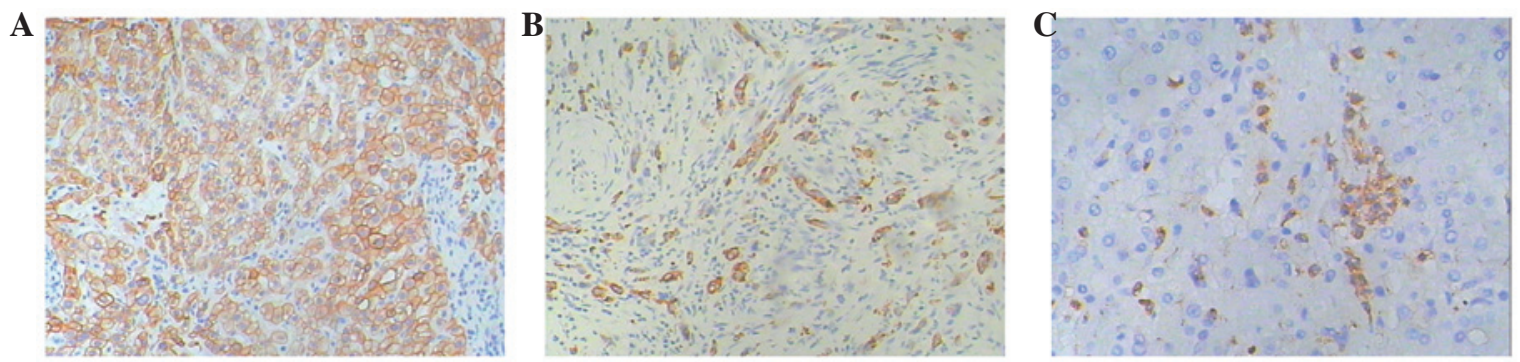

Figure 3. Immunohistochemistry results for (A) CK18(+); (B) CK19(++); (C) pCEA(+++). Magnification, x100.
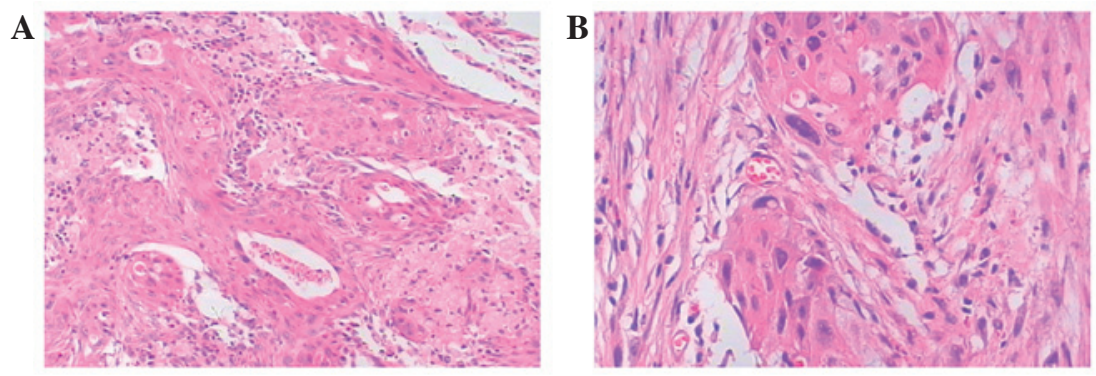

Figure 4. Microscopically, infiltration of poorly differentiated carcinoma tissue in the proliferative fibrous tissue and interaction between foam cells and multinucleated giant cells in the mesenchyme were observed. H\&E staining. (A) Magnification, x100; (B) magnification, x200. H\&E hematoxylin and eosin.
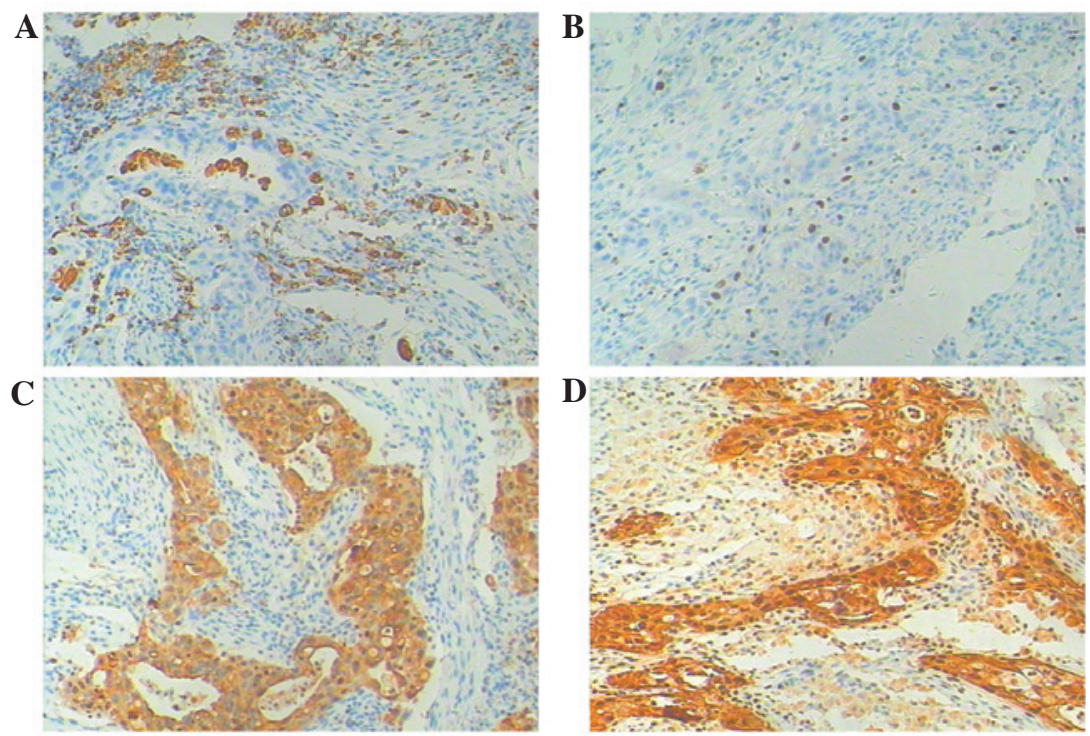

Figure 5. Immunohistochemistry results for (A) CD68(-); (B) Ki-67(+,<25\%); (C) CK(+); (D) CK5(+). Magnification, x100. 
Currently, the definitive diagnosis mainly relies on pathology and immunohistochemistry.

In cases of liver primary squamous carcinoma, due to its high malignancy, poor prognosis and late definitive diagnosis, survival is generally no longer than 1 year (11). As the incidence of liver primary squamous carcinoma is extremely low, its therapeutic regime has not been agreed. Reported therapeutic methods include surgical resection, generalized chemotherapy, radiotherapy, hepatic arterial chemoembolization (HACE) and combinations of these therapies. In the present study, the patient underwent liver tumor radiofrequency ablation, but the patient's abdominal pain continued after the surgery. As the patient's physical condition was too weak to tolerate generalized chemotherapy, local radiotherapy and supportive therapy is being carried out, and the patient's pain has already been relieved. The current viewpoint is that HACE combined with radiotherapy or generalized chemotherapy may extend survival in patients with primary squamous carcinoma, whereas the timely total surgical resection of tumor is the key to preventing invasive tumor growth and further surgical resection, systematic generalized chemotherapy or HACE may be used for patients with postoperative recurrence of the disease. We determined that local radiotherapy may be used to relieve symptoms in patients who are unable to undergo resection of the tumor or tolerate generalized chemotherapy.

\section{Acknowledgements}

This study was financially supported by grantno.2011CB504302 from the 973 Program of China and grant nos. ZR2010HL018 and Y2008C30 from the Natural Science Foundation of Shandong. We appreciate the valuable comments from other members of our laboratories.

\section{References}

1. Imai T: Ein Fall von zystischem Teratom der Leber, in welchem Plattenepithelkrebs entstand. Trans Soc Pathol Jap 24: 578-580, 1934 (In German).

2. Lynch MJ, McLeod MK, Weatherbee L, Gilsdorf JR, Guice KS and Eckhauser FE: Squamous cell cancer of the liver arising from a solitary benign nonparasitic hepatic cyst. Am J Gastroenterol 83: 426-431, 1988.

3. Banbury J, Conlon KC, Ghossein R and Brennan MF: Primary squamous cell carcinoma within a solitary nonparasitic hepatic cyst. J Surgl Oncol 57: 210-212, 1994.

4. Monteagudo M, Vidal G, Moreno M, et al: Squamous cell carcinoma and infection in a solitary hepatic cyst. Eur J Gastroenterol Hepatol 10: 1051-1053, 1998.

5. Vick DJ, Goodman ZD and Ishak KG: Squamous cell carcinoma arising in a ciliated hepatic foregut cyst. Arch Pathol Lab Med 123: 1115-1117, 1999.

6. Furlanetto A and Dei Tos AP: Squamous cell carcinoma arising in a ciliated hepatic foregut cyst. Virchows Archiv 441: 296-298, 2002.

7. Yagi H, Ueda M, Kawachi S, et al: Squamous cell carcinoma of the liver originating from non-parasitic cysts after a 15 year follow-up. Eur J Gastroenterol Hepatol 16: 1051-1056, 2004.

8. Zhang X, Wang Z and Dong Y: Squamous cell carcinoma arising in a ciliated hepatic foregut cyst: case report and literature review. Pathol Res Pract 205: 498-501, 2009.

9. Song E, Kew MC, Grieve T, Isaacson C and Myburgh JA: Primary squamous cell carcinoma of the liver occurring in association with hepatolithiasis. Cancer 53: 542-546, 1984.

10. Arase Y, Endo Y, Hara M, Kumada H, Ikeda K and Yoshiba A: Hepatic squamous cell carcinoma with hypercalcemia in liver cirrhosis. Acta Pathol Jpn 38: 643-650, 1988.

11. Yuki N, Hijikata Y, Kato M, Kawahara K and Wakasa K: Squamous cell carcinoma as a rare entity of primary liver tumor with grave prognosis. Hepatol Res 36: 322-327, 2006.

12. Spaggiari M, Di Benedetto F, Ballarin R, et al: Primary squamous cell carcinoma of the liver associated with Caroli's disease: a case report. Onkologie 34: 193-195, 2011.

13. Kaji R, Sasaki N, Tateishi I, et al: A case report of primary hepatic squamous cell carcinoma that remarkably responded to low dose arterial injection of anti-cancer drugs. Kurume Med J 50: 71-75, 2003. 